\title{
Investigación \\ MEJORAMIENTO DE PLANTAS NATIVAS SUDAMERICANAS: ALTAS TEMPERATURAS COMO HERRAMIENTA PARA SUPERAR BARRERAS PRECIGÓTICAS EN EL COMPLEJO Calibrachoa-Nierembergia
}

\section{Research \\ IMPROVEMENT OF NATIVE SOUTH AMERICAN PLANTS: HIGH TEMPERATURES AS A TOOL TO OVERCOME PRECIGOTIC BARRIERS IN THE COMPLEX Calibrachoa-Nierembergia}

\author{
Víctor José Milicia ${ }^{1}$, Andrea Coviella ${ }^{2}$, María Silvina Soto $^{2}$, y Ángel Chiesa ${ }^{1}$ \\ ${ }^{1}$ Facultad de Ciencias Agrarias, Universidad Nacional de Lomas de Zamora, Ruta Provincial $\mathrm{N}^{\circ} 4, \mathrm{~km}$ \\ 2, C.P. (1832), Buenos Aires, Argentina. \\ ${ }^{2}$ Instituto de Floricultura, Centro de Investigación de Recursos Naturales (CIRN) - Instituto Nacional de \\ Tecnología Agropecuaria (INTA), Nicolas Repetto y de los Reseros s/n (1686), Hurlingham, Argentina. \\ * Autor para correspondencia E-mail: victormilicia@hotmail.com
}

\section{RESUMEN}

El objetivo del presente trabajo fue evaluar la utilización de altas temperaturas como metodología para superar las barreras de incompatibilidad precigótica en cruzamientos interespecíficos entre especies del género Calibrachoa y Nierembergia. Se realizaron los cruzamientos en las combinaciones $N$. scoparia $\times$ C. ovalifolia, $N$. scopaira $\times$ C. caesia, $N$. linariaefolia $\times$ C. thymifolia y $N$. linariaefolia $x$ C. ovalifolia, utilizando diferentes genotipos. Las plantas con los botones florales preparados para polinizar fueron colocadas en estufa a $50^{\circ} \mathrm{C}$ durante 5 minutos para $N$. scoparia y 2,5 minutos para $N$. linariaefolia. Cumplido el tiempo se retiraron de la estufa y se polinizaron con polen fresco de Calibrachoa spp. Se evaluó el porcentaje de cruzamientos con llegada de tubos polínicos al estilo y al ovario. La aplicación de altas temperaturas a gineceos de $N$. scoparia se presentó como una metodología exitosa en la combinación $N$. scoparia x C. caesia, debido a que en todos los casos la cantidad de tubos polínicos en ovario fue mayor al testigo. En el caso de N. linariaefolia los resultados muestran que si bien hay una mejora con este método, no hubo un incremento de tubos polínicos en ovarios. Los resultados de este trabajo permitieron generar nuevos conocimientos sobre las relaciones intergenéricas, las barreras de incompatibilidad y aspectos vinculados con la biología floral, que sentarán las bases para la obtención de híbridos.

Palabras clave: Solanaceae, hibridación intergenérica, tubo polínico, incompatibilidad

\section{ABSTRACT}

The aim of this study was to evaluate the use of high temperatures to overcome prezygotic interspecific incompatibility barriers in crosses between species of Calibrachoa and Nierembergia. The following combinations were evaluated: N. scoparia $x$ C. ovalifolia, N. scoparia $x$ C. caesia, $N$. linariaefolia $x C$. thymifolia and N. linariaefolia $x$ C. ovalifolia, using different genotypes. The plants with prepared buds were placed in a stove at $50^{\circ} \mathrm{C}$ for 5 minutes for $N$. scoparia and 2.5 minutes for N. linariaefolia. Subsequently, the plants are removed from the oven and pollinated with fresh pollen. The percentage of crosses of pollen tubes to the style and ovary was evaluated. Application 
of high temperatures in N. scoparia could be a good approach in the combination N. scoparia $x$ C. caesia, because in all cases the amount of pollen tubes in ovary was higher than in the control. For $N$. linariaefolia results show that, although there is an improvement with this method, no increase of pollen tubes which reached the ovaries. The results of this work allowed generate new knowledge about genera relationships, incompatibility barriers and issues related to the floral biology, which are basic for obtaining hybrids.

Key words: Solanaceae, intergeneric hybrid, pollen tubes, incompatibility.

\section{INTRODUCCION}

Muchos géneros originarios de América del Sur, tales como Petunia, Glandularia, Begonia y Alstroemeria, han contribuido al desarrollo de variedades comerciales ornamentales en el mundo. Sin embargo, hasta el momento los países de este continente no han explotado el uso de los recursos genéticos con valor ornamental. Desde 1999, en un intento de revertir esta situación, el Instituto de Floricultura perteneciente al Instituto Nacional de Tecnología Agropecuaria (INTA) de la Argentina, comenzó actividades de investigación para desarrollar variedades ornamentales a partir de plantas nativas. Estas abarcaron desde la recolección de germoplasma hasta la domesticación y mejoramiento en géneros promisorios (Facciuto et al., 2008). En este marco una de las herramientas más utilizadas, que a su vez se presenta como la más importantes en el mejoramiento de plantas ornamentales, es la hibridación interespecífica, que posibilita obtener variación en caracteres que en las especies originales no se expresan (Van Tuyl, 2003).

En efecto, los cruzamientos interespecíficos fueron herramientas básicas en programas de mejoramiento de cultivos. El género Lilium constituye uno de los ejemplos de mayor importancia, contribuyendo a la obtención de cruzamientos entre Lilium longiflorum Thunb. y Lilium asiáticos. Esto no sólo permitió introducir variantes deseables de algunos caracteres de numerosas especies en cultivares comerciales, sino también la expansión del conocimiento en la relaciones entre las especies y el estudio de los mecanismos de introgresión génica (Van Tuyl et al., 1986; Lim et al., 2008).

La obtención de híbridos interespecíficos y, en mayor medida, intergenéricos, muchas veces está limitada por diferentes barreras de incompatibilidad (Nettancourt, 2005), las cuales han sido identificadas y superadas después, produciendo germoplasma mejorado (Dinu et al., 2005). Las barreras de incompatibilidad se establecen antes o después de la fecundación y se presentan en diversas formas, según los géneros, y en función de estas se seleccionan las diferentes técnicas utilizadas para superarlas.

Dentro de la familia Solanaceae el complejo
Calibrachoa-Nierembergia presenta características sobresalientes como recursos fitogenéticos para la obtención de variedades ornamentales. El género Calibrachoa presenta numerosas variedades de gran valor ornamental, principalmente por la variabilidad que presentan en color y tamaño de flores (Griesbach, 2006). Por otra parte, el género Nierembergia, tiene escasa variabilidad en cuanto a forma y color de flor, pero presenta un excelente comportamiento en condiciones de cultivo, con baja susceptibilidad a plagas y enfermedades (Soto, 2007). Esta aptitud lo convierte en un género atractivo para ser utilizado como fuente génica para traspasar estas características hacia otros géneros, como Calibrachoa.

En función de la necesidad de conjugar caracteres interesantes de ambos géneros para la obtención de variedades ornamentales, se comenzó a estudiar la compatibilidad en el complejo Calibrachoa-Nierembergia, en que el estado actual del conocimiento en la familia Solanaceae explica la existencia de un sistema de autoincompatibilidad gametofítica, donde la germinación del polen en el estigma y la elongación del tubo polínico, en la mayoría de los casos son normales, produciéndose una inhibición más tardía a lo largo de su desarrollo por el estilo (Dowd et al., 2000). En Solanaceae el locus $S$ codifica para una glicoproteína del estilo (SLG: Style Locus Glycoprotein), la cual es secretada al espacio extracelular del mismo (Cheung et al., 2000). Se propone que estas proteínas actúan como agente citotóxico S-específico degradando los tubos polínicos incompatibles (Huang et al., 1994). Teniendo en cuenta que el modelo de auto-incompatibilidad gametofítica propone que la incompatibilidad se expresa a nivel del estilo (Pandey, 1969; Nettancourt, 2005), se podría asumir que procesos semejantes pudieran regir la incompatibilidad entre diferentes géneros, como en los casos presentados en este trabajo (Liedl y Anderson, 1994).

El presente trabajo tuvo como objetivo evaluar la utilización de altas temperaturas previas a la polinización sobre gineceos, como metodología para superar barreras de incompatibilidad precigóticas en el complejo Calibrachoa-Nierembergia. La hipótesis es que la desnaturalización de proteínas vinculadas a las barreras de incompatibilidad reduce o anula su función. 


\section{MATERIALES Y MÉTODOS}

Los ensayos se realizaron en el Instituto de Floricultura del Instituto Nacional de Tecnología

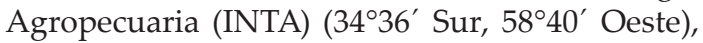
Castelar, Argentina, durante la primavera del año 2013.

Para los estudios de compatibilidad se utilizaron dos especies correspondientes al género Nierembergia: N. linariaefolia Graham. y N. scoparia Sendth., y tres especies correspondientes al género Calibrachoa: C. ovalifolia (Miers) Stehmann \& Semir (dos genotipos), C. thymifolia (A. St.-Hill.) Stehmann \& Semir (ocho genotipos), y C. caesia (Sendth.) Wijsmann (cuatro genotipos) provenientes de distintas zonas de la mesopotamia argentina.

Las plantas parentales fueron cultivadas en macetas de 3 litros de capacidad, utilizando un sustrato compuesto por hoja de pino:turba subtropical:tierra (2:1:1 v:v:v), bajo condiciones de invernáculo de plástico durante todo el ensayo. El manejo sanitario se realizó de la siguiente manera: una pulverización cada 15 días utilizando según las necesidades, fungicidas (carbendazim, captan, procimidone, azoxistrobina, cyproconazole, miclobutanilo), acaricidas (clofentezine) e insecticidas (buprofezin, fenitrothion, mercaptotion, clorpirifos, cartap, metomilo, fenarimol, imidacloprid, abamectina). La utilización de cada producto fue alternada. También se efectuó una fertilización sistemática con NPK 15:10:15, aplicando una dosis de $150 \mathrm{mg} \mathrm{kg}^{-1}$ de $\mathrm{N}$ una vez por semana, utilizando fertirriego con dosificadores hidráulicos.

\section{Determinación del tiempo de exposición de gineceos a alta temperatura}

Para evaluar los diferentes tratamientos de temperaturas a las que se someten los gineceos previa a la polinización, primero se determinó el tiempo máximo de exposición de los mismos a $50^{\circ} \mathrm{C} \sin$ que se produzca necrosis en el tejido. Para tal fin se colocaron las macetas en estufa a $50^{\circ} \mathrm{C}$ durante distintos tiempos de prueba: 20, 10, 5 y 2,5 minutos. Para los tratamientos se seleccionaron aquellos tiempos de exposición a $50^{\circ} \mathrm{C}$ en los cuales no se observó necrosis del gineceo para cada especie.

\section{Evaluación de altas temperaturas como método para superar barreras de incompatibilidad}

Se seleccionaron 10 botones florales por genotipo en estado de desarrollo previo a la antesis con una longitud entre 2 y $2,5 \mathrm{~cm}$ para $N$. linariaefolia, y entre 1,8 y $2 \mathrm{~cm}$ para $N$. scoparia para realizar los cruzamientos. A estos botones florales se les retiró la porción superior de los pé- talos y los estambres, dejando libre el estigma. Posteriormente las macetas fueron colocadas en estufa a $50^{\circ} \mathrm{C}$ durante 5 minutos para $N$. scoparia conformando el tratamiento número 1 (T1) y 2,5 minutos para $N$. linariaefolia conformando el tratamiento número 2 (T2). Cumplido el tiempo se retiraron de la estufa y se polinizaron con polen fresco de la especie putativa paterna Calibrachoa spp.

Como testigo (T0) se realizaron idénticos cruzamientos pero sin la exposición de las plantas a altas temperaturas. Las combinaciones testigo fueron realizadas con granos de polen fresco de los distintos genotipos de la misma especie usada como parental masculino, y con los que se polinizó cada estigma del parental femenino testigo. Después de $24 \mathrm{~h}$ se recolectaron los gineceos y se fijaron en FAA (etanol 50\% : agua destilada : formaldehido : acético glacial, 10:7:2:1, v:v:v:v) para su posterior observación.

Para realizar las observaciones al microscopio, el tejido fue previamente hidrolizado con una solución acuosa de $\mathrm{NaOH} \mathrm{0,6} \mathrm{N}$ durante una noche. Seguidamente, el material fue sumergido en azul de anilina $0,1 \%$ solución acuosa para su posterior observación con microscopio de epifluorescencia (OLYMPUS, modelo BX50F4, Tokio, Japón) con luz ultra violeta de excitación de 365 a $400 \mathrm{~nm}$ de longitud de onda (Martin, 1959). Se evaluó el desarrollo de los tubos polínicos en gineceos, considerando la distancia recorrida y el porcentaje de cruzamientos mediante el número de ovarios en los cuales los tubos polínicos llegaron al ovario. Las fotos se tomaron con una cámara Olympus DP 72.

Para cada tratamiento se realizaron 10 repeticiones, realizando en total 200 cruzamientos.

Para el análisis estadístico se utilizó la prueba de diferencia de proporciones basadas en la distribución exacta del estadístico de Fisher, según corresponde con el paquete estadístico Infostat (Di Rienzo et al., 2008). Las pruebas estadísticas que mostraron valor $\mathrm{P}<0,05$ fueron consideradas como estadísticamente significativas.

\section{RESULTADOS}

\section{Determinación del tiempo de exposición a altas temperaturas}

En los distintos tiempos de exposición se observó la presencia de necrosis en los tejidos del gineceo luego de pasadas las $24 \mathrm{~h}$. Por lo tanto, a partir de la presencia de necrosis en los gineceos sometidos a los diferentes tratamientos de temperatura, se seleccionaron como tiempo límite 5 minutos de exposición a $50^{\circ} \mathrm{C}$ para $N$. scoparia y 2,5 minutos a $50^{\circ} \mathrm{C}$ para $N$. linariaefolia (Tabla 1 ) 
Tabla 1. Necrosis en gineceos de N. scoparia y N. linariaefolia después de exponerlos a $50^{\circ} \mathrm{C}$ durante 20, 10, 5 y 2,5 minutos.

Table 1. N. scoparia and N. linariaefolia ginoecia necrosis after exposition at $50^{\circ} \mathrm{C}$ for $20,10,5$ and 2.5 minutes.

\begin{tabular}{|c|c|c|c|c|}
\hline & \multicolumn{4}{|c|}{ Tiempo de exposición } \\
\hline & 20 & 10 & 5 & 2,5 \\
\hline N. scoparia & Tejido necrosado & Tejido necrosado & Tejido sano & Tejido sano \\
\hline N. linariaefolia & Tejido necrosado & Tejido necrosado & Tejido necrosado & Tejido sano \\
\hline
\end{tabular}

Evaluación de altas temperaturas como método para superar barreras de incompatibilidad.

A continuación se exponen los resultados agrupados por técnica y por parentales femeninos.

Exposición de los gineceos de N. scoparia a $50^{\circ} \mathrm{C}$ durante 5 minutos

N. scoparia (+): en la combinación N. scoparia x C. ovalifolia la germinación de los granos de polen se registró en todos los estigmas polinizados para los genotipos de C. ovalifolia evaluados. El desarrollo de los tubos polínicos fue normal, con dirección hacia el ovario, y con presencia de tapones de calosa para el genotipo 2 (Fig. 1), mientras que en el genotipo 1 se observó calosa dispersa sobre la pared del tubo polínico y en muy pocos casos la formación de tapones. Con respecto a la llegada de tubos polínicos a los estilos (Fig. 2), se observó en el $88 \%$ de los estilos para el genotipo 2 , mientras que para el genotipo 1 en el $40 \%$. En el mismo sentido, el genotipo 2 presentó tubos polínicos que alcanzaron el ovario en el $44 \%$ de los cruzamientos, presentando diferencias significativas $(\mathrm{P}<0,05)$ con el testigo $(5,8 \%)$, mientras que en el genotipo 1 los tubos polínicos no alcanzaron el ovario (Tabla 2). Como testigo se utilizó el cruzamiento pool de $N$. scoparia $\times$ C. ovalifolia con granos de polen de sus dos genotipos.

En los cruzamientos realizados entre N. scoparia y C. caesia se observó germinación normal de los granos de polen, los tubos polínicos presentaron tapones de calosa y dirección hacia el ovario, para los 4 genotipos de $C$. caesia evaluados (Fig. 3). La presencia de tubo polínico en los estilos en estos cruzamientos (Fig. 4) varió entre un $88 \%$ a $50 \%$ (Tabla 2) según el genotipo usado como donante de polen, sin presentar diferencias significativas entre ellos ni con el testigo $(57,1 \%)$. Como testigo se utilizó el cruzamiento pool de $N$. scoparia $\times$ C. caesia con granos de polen de sus cuatro genotipos. Con respecto a la llegada de tubos polínicos al ovario (Fig. 5), esto varió según los genotipos, siendo de $50 \%$ hasta $71 \%$ (Tabla 2 ). Cabe destacar que si bien estadísticamente no se presentaron diferencias significativas $(\mathrm{P} \geq 0,05)$ con el testigo (25\%) para el análisis estadístico utilizado, el tratamiento de temperatura incrementó el porcentaje de llegada al ovario para todos los genotipos.

\section{Exposición de los gineceos de $N$. linariaefolia a} $50^{\circ} \mathrm{C}$ durante 2,5 minutos

En la combinación N. linariaefolia ( $(+) \times C$. ovalifolia se observó germinación normal de los granos de polen, con formación de tapones de calosa y dirección hacia el ovario para los 2 genotipos de C. ovalifolia evaluados (Fig. 6). El desarrollo de tubos polínicos a los estilos de estos cruzamientos difirió entre los distintos genotipos, siendo 100\% para el genotipo 2 y $25 \%$ en los cruzamientos del genotipo 1 (Tabla 2). Por otra parte, en ninguno de los cruzamientos se detectó la llegada del tubo polínico al ovario (Tabla 2 ).

La combinación $N$. linariaefolia $x$ C. thymifolia mostró germinación normal de los granos de polen, formación de tapones de calosa y desarrollo del tubo polínico hacia el ovario para los 8 genotipos de C. thymifolia. El desarrollo de los tubos polínicos por los estilos (Fig. 7) presentó porcentajes variables para los distintos genotipos, como se puede observar en la Tabla 2. Todos los genotipos de $C$. thymifolia presentaron diferencias significativas con el testigo con excepción del genotipo 3 . Como testigo se utilizó el cruzamiento pool de $N$. linariaefolia $\times$ C. thymifolia con granos de polen de sus ocho genotipos. En lo que se refiere al porcentaje de llegada al ovario, sólo el genotipo 7 presentó desarrollo de tubos polínicos hasta el ovario, sin diferencias estadísticamente significativas con el testigo $(0 \%)$ (Tabla 2$)$.

\section{DISCUSIÓN}

Muchos autores indican la importancia de la temperatura como factor físico que influye sobre la incompatibilidad (Fett et al., 1976; Van Tuyl y De Jeu, 2005). Cuando las proteínas termolábiles SLG son sometidas a altas temperaturas $\left(50^{\circ} \mathrm{C}\right)$ se desnaturalizan y no cumplen su función, permi- 


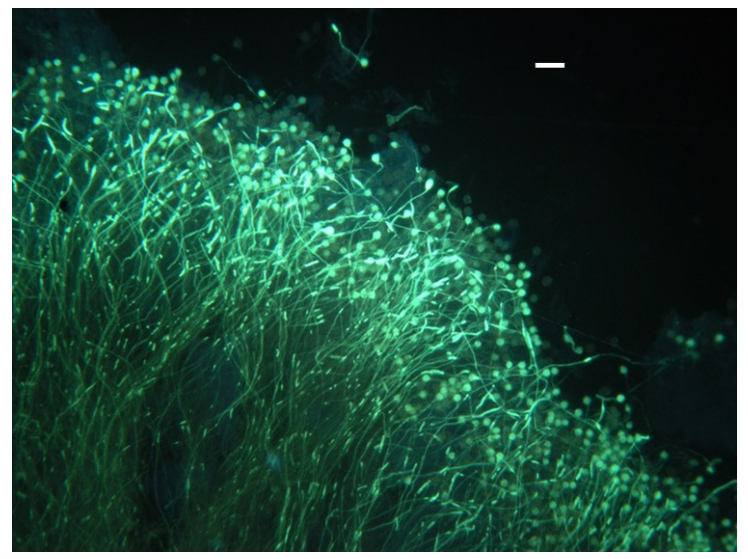

Fig. 1. Germinación de granos de polen de C. ovalifolia sobre el estigma de N. scoparia. Barra $=\mathbf{1 0 0} \mu \mathrm{m}$

Fig. 1. Germination of pollen grains of C. ovalifolia on stigma of N. scoparia.

Bar $=\mathbf{1 0 0} \mu \mathrm{m}$

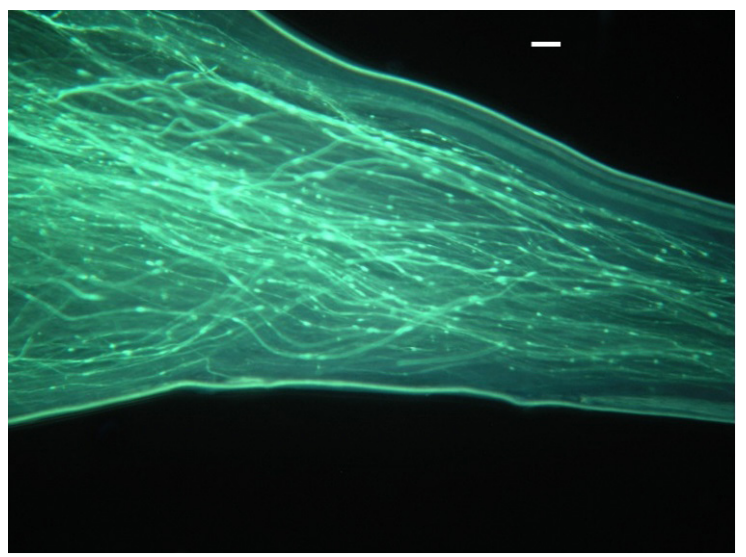

Fig. 2. Tubos polínicos de C. ovalifolia en estilo de N. scoparia a las 24 horas postpolinización. Barra $=100 \mu \mathrm{m}$

Fig. 2. Pollen tubes of $C$. ovalifolia in style $N$. scoparia 24 hours postpolinización. Bar $=\mathbf{1 0 0} \mu \mathrm{m}$

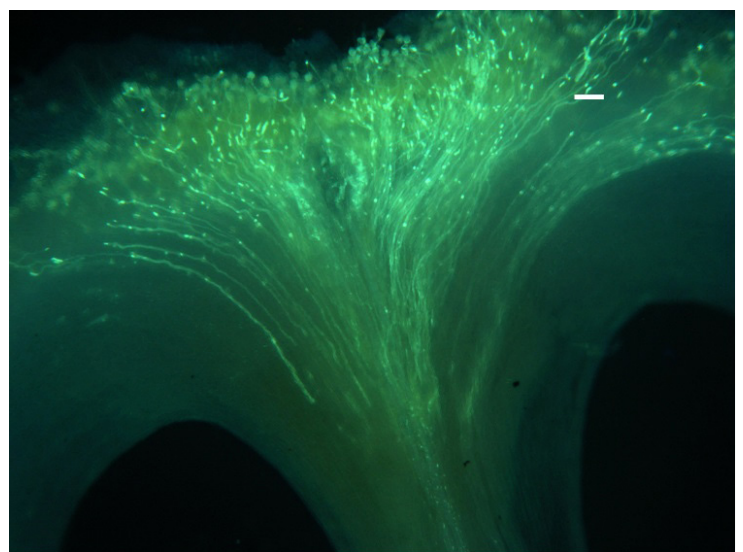

Fig. 3. Germinación de granos de polen de C. caesia sobre estigma de N. scoparia. Barra $=\mathbf{1 0 0} \mu \mathrm{m}$

Fig. 3. Germination of pollen grains of $C$. caesia on stigma of $N$. scoparia. Bar $=100 \mu \mathrm{m}$ 
Tabla 2. Presencia de tubos polínicos en estilo y ovario en cruzamientos intergenéricos entre $N$. scoparia o N. linariaefolia como parental femenino cruzadas con C. ovalifolia, C. caesia, C. thymifolia y $C$. ovalifolia como parental masculino, respectivamente, 24 h postpolinización, utilizando como técnica para superar barreras precígoticas exposición durante 5 y 2,5 minutos a $50^{\circ} \mathrm{C}$.

Table 2. Pollen tubes in the style and ovary in intergeneric crosses between $N$. scoparia and $N$. linariaefolia as female parent crossed with C. ovalifolia, C. caesia, C. thymifolia and C. ovalifolia as male parent, respectively, after 24 hours postpolinization, using as a technique to overcome barriers prezygotic exposure for 5 (T1) and 2.5 (T2) minutes at $50^{\circ} \mathrm{C}$, and (T0) without exposure at $50^{\circ} \mathrm{C}$.

\begin{tabular}{|c|c|c|c|}
\hline Cruzamientos & $\begin{array}{l}\text { Tratamiento } \\
\text { a } 50^{\circ} \mathrm{C} \text { por }\end{array}$ & $\begin{array}{l}\text { Cruzamientos } \\
\text { con tubos } \\
\text { polínicos en } \\
\text { estilo (\%) }\end{array}$ & $\begin{array}{c}\text { Cruzamientos } \\
\text { con tubos } \\
\text { polínicos en } \\
\text { ovario }(\%)\end{array}$ \\
\hline N. scoparia $x$ C. ovalifolia (genotipo $1 \times$ genotipo 1 ) & $\mathrm{T} 15 \mathrm{~min}$ & $40 \mathrm{a}$ & $0 \mathrm{a}$ \\
\hline N. scoparia $x$ C. ovalifolia (genotipo $1 \times$ genotipo 2 ) & $\mathrm{T} 25 \mathrm{~min}$ & $88,8 \mathrm{~b}$ & $44,4 \mathrm{~b}$ \\
\hline N. scoparia $x$ C. ovalifolia (testigo) & T0 0 min & $85 \mathrm{~b}$ & 5,8 a \\
\hline N. scoparia $x$ C. caesia (genotipo $1 \times$ genotipo 1 ) & $\mathrm{T} 15 \mathrm{~min}$ & $80 \mathrm{a}$ & 66,66 a \\
\hline N. scoparia $x$ C. caesia (genotipo 1 x genotipo 2) & $\mathrm{T} 25 \mathrm{~min}$ & 88,8 a & $50,00 \mathrm{a}$ \\
\hline N. scoparia $x$ C. caesia (genotipo $1 \times$ genotipo 3 ) & $\mathrm{T} 35 \mathrm{~min}$ & 77,7 a & $60 \mathrm{a}$ \\
\hline N. scoparia $x$ C. caesia (genotipo $1 \times$ genotipo 4 ) & $\mathrm{T} 45 \mathrm{~min}$ & $50 \mathrm{a}$ & $71,4 \mathrm{a}$ \\
\hline N. scoparia $x$ C. caesia (testigo) & T $00 \mathrm{~min}$ & 57,1 a & 25 a \\
\hline N. linariaefolia $x$ C. ovalifolia (genotipo $1 \times$ genotipo1) & $\mathrm{T} 22,5 \mathrm{~min}$ & $25,0 \mathrm{a}$ & $0 \mathrm{a}$ \\
\hline N. linariaefolia $x$ C. ovalifolia (genotipo $1 \times$ genotipo2) & $\mathrm{T} 22,5 \mathrm{~min}$ & $100,0 \mathrm{~b}$ & $0 \mathrm{a}$ \\
\hline N. linariaefolia $x$ C. ovalifolia (testigo) & T0 $0 \mathrm{~min}$ & $90,4 \mathrm{~b}$ & 0 a \\
\hline N. linariaefolia $x$ C. thymifolia (genotipo $1 \times$ genotipo 1 ) & $\mathrm{T} 22,5 \mathrm{~min}$ & $87,5 \mathrm{~b}$ & $0 \mathrm{a}$ \\
\hline N. linariaefolia $x$ C. thymifolia (genotipo $1 \times$ genotipo 2 ) & $\mathrm{T} 22,5 \mathrm{~min}$ & $90,0 \mathrm{~b}$ & $0 \mathrm{a}$ \\
\hline N. linariaefolia $x$ C. thymifolia (genotipo $1 \times$ genotipo 3 ) & $\mathrm{T} 22,5 \mathrm{~min}$ & 33,3 a & $0 \mathrm{a}$ \\
\hline N. linariaefolia $x$ C. thymifolia (genotipo $1 \times$ genotipo 4 ) & $\mathrm{T} 22,5 \mathrm{~min}$ & $100,0 \mathrm{~b}$ & $0 \mathrm{a}$ \\
\hline N. linariaefolia $x$ C. thymifolia (genotipo $1 \times$ genotipo 5) & $\mathrm{T} 22,5 \mathrm{~min}$ & $80,0 \mathrm{~b}$ & $0 \mathrm{a}$ \\
\hline N. linariaefolia $x$ C. thymifolia (genotipo $1 \times$ genotipo 6) & $\mathrm{T} 22,5 \mathrm{~min}$ & $70,0 \mathrm{~b}$ & $0 \mathrm{a}$ \\
\hline N. linariaefolia $x$ C. thymifolia (genotipo $1 \times$ genotipo 7 ) & $\mathrm{T} 22,5 \mathrm{~min}$ & $90,0 \mathrm{~b}$ & $11,1 \mathrm{a}$ \\
\hline N. linariaefolia $x$ C. thymifolia (genotipo $1 \times$ genotipo 8 ) & $\mathrm{T} 22,5 \mathrm{~min}$ & $100,0 \mathrm{~b}$ & $0 \mathrm{a}$ \\
\hline N. linariaefolia $x$ C. thymifolia (testigo) & T0 $0 \mathrm{~min}$ & 23,5 a & 0 a \\
\hline
\end{tabular}

Letras diferentes indican diferencias significativas según análisis de proporciones por el estadístico Fisher $(\mathrm{p}<0,05)$

tiendo el desarrollo de los tubos polínicos en cruzamientos incompatibles (Roberts et al., 1979; Van Tuyl y De Jeu, 2005). Tratamientos a altas temperaturas $\left(50^{\circ} \mathrm{C}\right)$ aplicados a pistilos previo a la polinización permiten superar barreras de incompatibilidad (Matsubara, 1980; Sharma, 2009). Entre otras especies en que se usaron distintos valores de temperatura se pueden destacar Raphanus sativus L. entre 30 y $50^{\circ} \mathrm{C}$ (Matsubara, 1980), y Tulipa gesneriana L. a $50^{\circ} \mathrm{C}$ (Okazaki y Murakami, 1992). Estos resultados son comparables con los obtenidos en los cruzamientos intergenéricos entre $N$. scoparia y los cuatro genotipos de C. caesia, donde la exposición durante cinco minutos a $50^{\circ} \mathrm{C}$ permitió incrementar el desarrollo de tubos polínicos de C. caesia hasta los ovarios de $N$. scoparia. .

En los cruzamientos entre $N$. scoparia y N. linariaefolia con los dos genotipos de C. ovalifolia, los resultados mostraron diferencia $(P<0,05)$ entre combinaciones. En este caso hubo una respuesta diferente entre genotipos. En hibridaciones realizadas entre los géneros Brassica y Crambe, Wang et al. (1996) también detectaron resultados variables al utilizar diferentes genotipos.

Por otro lado, en los cruzamientos con N. linariaefolia y $C$. thymifolia aumentó significativamen- 


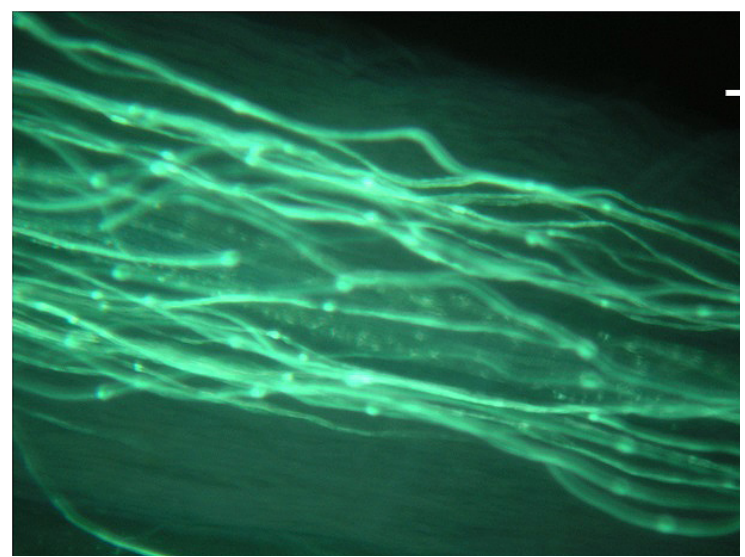

Fig. 4. Tubos polínicos de C. caesia en estilos de N. scoparia a las 24 horas postpolinización. Barra $=100 \mu \mathrm{m}$

Fig. 4. Pollen tubes of $C$. caesia in style of $N$. scoparia 24 hours postpolinización.

Bar $=100 \mu \mathrm{m}$

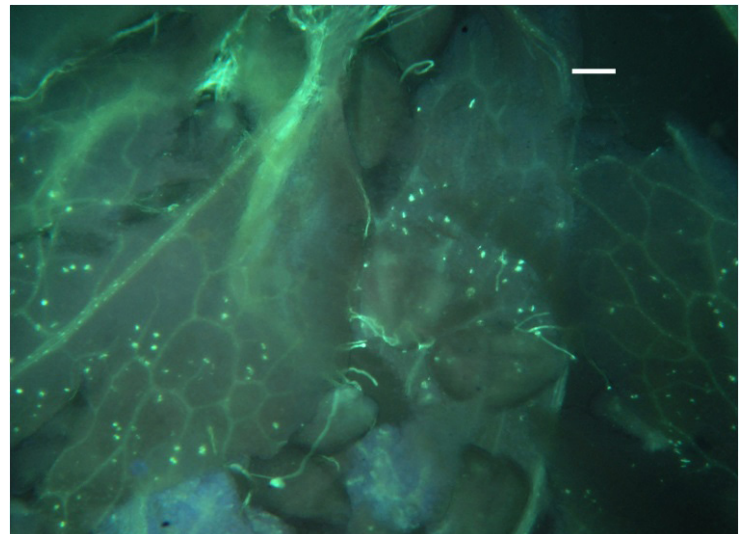

Fig. 5. Tubos polínicos de $C$. caesia penetrando la micrópila de los óvulos de $N$. scoparia en cruzamientos intergenéricos 24 horas postpolinización.

Barra $=\mathbf{1 0 0} \mu \mathrm{m}$

Fig. 5. Pollen tubes of $C$. caesia going through the micropyle of ovules of $N$. scoparia in intergeneric crosses 24 hours postpolinización.

Bar $=100 \mu \mathrm{m}$

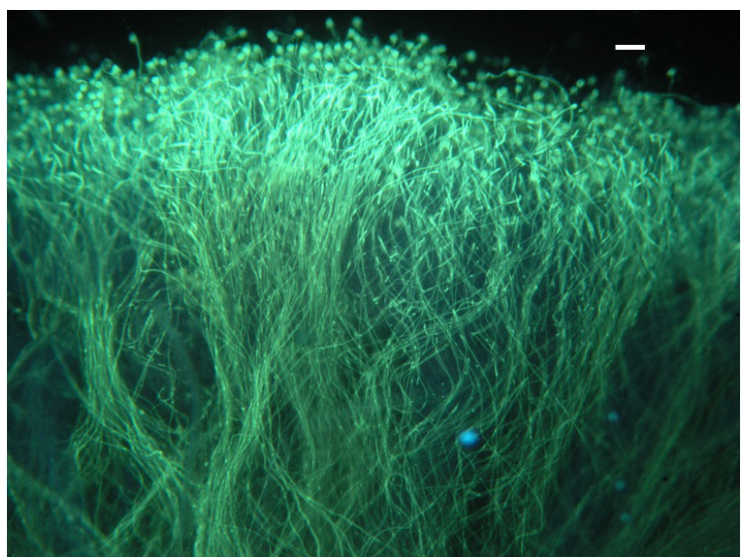

Fig. 6. Germinación de granos de polen de C. ovalifolia sobre estigma de N. linariaefolia. Barra $=100 \mu \mathrm{m}$

Fig. 6. Germination of pollen grains of C. ovalifolia in stigma of N. linariaefolia. Bar $=\mathbf{1 0 0} \mu \mathrm{m}$ 


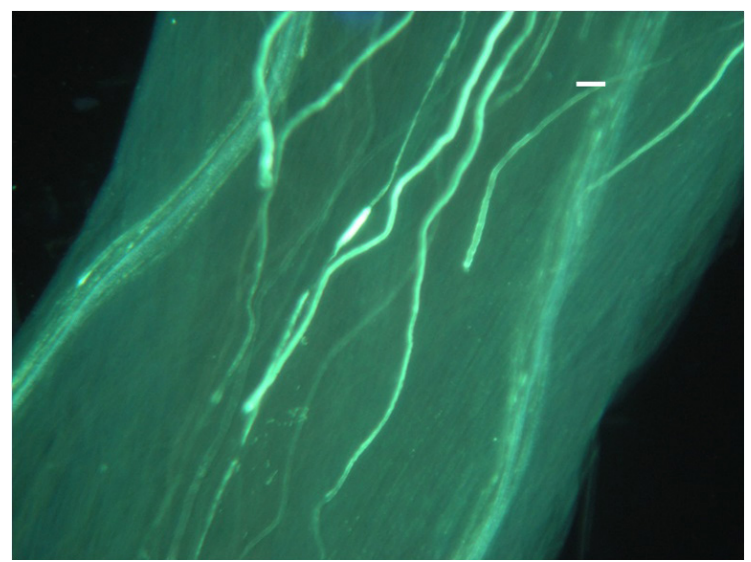

Fig. 7. Tubos polínicos de $C$. thymifolia en estilos de $N$. linariaefolia a las 24 horas postpolinización. Barra $=100 \mu \mathrm{m}$

Fig. 7. Pollen tubes of $C$. thymifolia in style of $N$. linariaefolia 24 hours postpolinización. Bar $=100 \mu \mathrm{m}$

te la presencia de tubos polínicos en los estilos de Nierembergia, a excepción del genotipo 3, pero no así la llegada de estos al ovario, donde sólo el genotipo 7 fue exitoso. Estos resultados podrían deberse a un tiempo post-polinización demasiado corto, ya que los gineceos fueron cosechados 24 horas después de polinizados, y posiblemente la llegada de los tubos polínicos requiera un tiempo mayor a 24 horas. O podría deberse a que la exposición a altas temperaturas no logró la completa desnaturalización de las proteínas.

Resultados similares fueron encontrados en investigaciones realizadas en Chaenomeles japonica (Sweet) Nak., especie auto-incompatible, donde en los cruzamientos incompatibles los tubos polínicos alcanzaron el ovario cuatro días más tarde que en los cruzamientos compatibles (Kaufmane y Rumpunen, 2002). Un fenómeno semejante podría darse en estos casos, donde los tubos polínicos requerirían 48 , 72 o más horas para alcanzar el ovario. Asimismo, Herrero y Dickinson (1981) encontraron en Petunia hybrida una disminución en la velocidad de desarrollo de los tubos polínicos en el estilo en cruzamientos incompatibles comparados con cruzamientos compatibles.

El presente estudio permitió generar nuevos conocimientos sobre las relaciones intergenéricas, las barreras de incompatibilidad y aspectos vinculados con la biología floral, que sentarán las bases para la obtención de híbridos a partir de plantas nativas latinoamericanas. Por otra parte, los resultados obtenidos utilizando técnicas para superar las barreras de incompatibilidad permitirán continuar con la búsqueda de nuevas alternativas y ajustes en las técnicas ya evaluadas para ser utilizadas en trabajos futuros.

\section{CONCLUSIONES}

La aplicación de altas temperaturas a gineceos de N. scoparia previo a la polinización es una metodología exitosa en la combinación $N$. scoparia $\mathrm{x}$ C. caesia, debido a que en todos los genotipos la cantidad de tubos polínicos que llegaron al ovario fue mayor al testigo.

En el caso de N. linariaefolia los resultados mostraron que si bien una temperatura alta mejora el desarrollo del tubo polínico a través del estilo, no hubo un incremento de tubos polínicos que alcanzaron el ovario con respecto al testigo.

\section{LITERATURA CITADA}

Cheung, A.Y., H.M. Wu, V. Di Stilio, R. Glaven, C. Chen, E. Wong, et al. 2000. Pollen-pistil interactions in Nicotiana tabacum. Ann. Bot. 85(A):29-37.

Dinu, I., R.J. Hayes, R.G. Kynast, R.L. Phillips, and C.A. Thill. 2005. Novel inter-series hybrids in Solanum, section Petota. Theor. Appl. Genet. 110:403-415.

Di Rienzo, J.A., F. Casanoves, M.G. Balzarini, L. Gonzalez, M. Tablada, y C.W. Robledo. 2008. InfoStat, versión 2008. Grupo InfoStat. FCA, Universidad Nacional de Córdoba, Córdoba, Argentina.

Dowd, P.E., A.G. Mc Cubbin, X. Wang, J.A. Verica, T. Tsukamoto, T. Ando, and T. Kao. 2000. Use of Petunia inflata as a model for the study of Solanaceae type self-incompatibility. Ann. Bot. 85:87-93

Facciuto, G., S. Soto, and S. Maldonado. 2008. Domestication and breeding of ornamental plants native to Argentina: The cases of Tabebuia and Nierembergia genera. Floriculture and Ornamental Biotechnology 5:164-170. 
Fett, W., D. Paxton, and D. Dickinson. 1976. Studies on the self-incompatibility response of Lilium longiflorum. Am. J. Bot. 63:1104-1108.

Griesbach, R.J. 2006. Petunia. p. 301-336. In Neil O. Anderson (ed.) Flower breeding and genetic. University of Minnesota, Minnesota, U.S.A.

Herrero, M., and H. Dickinson. 1981. Pollen tube development in Petunia hybrida following compatible and incompatible intraspecific matings. J. Cell Sci. 47:365-383.

Huang, S., H. Lee., B. Karunanandad, and T. Kao. 1994. Ribonuclease activity on Petunia inflata $S$ proteins is essential for rejection of self-pollen. The Plant Cell 6:1021-1028.

Kaufmane, E., and K. Rumpunen. 2002. Pollination, pollen tube growth and fertilization in Chaenomeles japonica (Japanese quince). Sci. Hort. 94:257-271.

Liedl, B., and N. Anderson. 1994. Reproductive barriers: Identification, uses and circumvention. p. 11-134. In Plant Breeding Reviews. John Wiley \& Sons, New York, USA.

Lim, K., R. Gonzalez, S. Zhou, M. Ramanna, J.M. Van Tuyl. 2008. Interspecific hibridization in Lily (Lilium): Taxonomic and Commercial Aspects of Using Species Hybrids in Breeding. p. 146-151. In Jaime A. Teixeira da Silva (ed.). Floriculture, Ornamental and Plant Biotechnology Advances and Topical Issues, Kagawa University, Japan.

Martin, R. N. 1959. Staining and observing pollen tubes in the style by means of fluorescence. Stain Technology 34:125-128.

Matsubara, S. 1980. Overcoming self-incompatibility in Raphanus satious L. with high temperature. J. Amer. Soc. Hort. Sci. 105:842-846.

Nettancourt De, D. 2005. Incompatibility and incongruity in wild and cultivated plants. Springer-Verlag, Berlin, Germany.
Okazaki, K., and K. Murakami. 1992. Effects of flowering time (in forcing culture), stigma excision, and high temperature on overcoming of self-incompatibility in tulip. J. Jap. Soc. Hortic. Sci. 61(2):405-411.

Pandey, K.K. 1969. Elements of the S-gene complex. V. Interspecific cross compatibility relationships in Lycopersicon. Proc. Natl. Acad. Sci. 79:5006-5010.

Roberts, I., A. Stead, D. Ockendon, and H. Dickinson. 1979. A glycoprotein associated with acquisition of the self-incompatibility system by maturing stigmas of Brassica oleracea. Planta 146:179-183.

Sharma, H.P. 2009. Plant embryology classical and experimental. Alpha Science International Ltd., London, United Kingdom. .

Soto, M.S. 2007. Estudios de las relaciones interespecíficas en el género Nierembergia, como herramientas del mejoramiento. Tesis Doctoral. Facultad de Ciencias Exactas y Naturales, Universidad de Buenos Aires, Argentina.

Van Tuyl, J.M., J. Franken, M.C. Jongerius, C.A.M. Lock, and A.A.M. Kwakkenbos. 1986. Interspecific hybridization in Lilium. Acta Hort. 177:591-595.

Van Tuyl, J.M., 2003. Interspecific hybridation and polyploidisation as tool in ornamental plant breeding. Acta Hort. 612:13-20.

Van Tuyl, J.M., De Jeu, J. 2005. Methods for overcoming interespecific crossing barriers. $p$. 1-30. In V.K. Shawnhey and K. R. Shivanna (eds.) Pollen biotechnology for crop. Production and improvement. Cambridge University Press, Cambridge, United Kingdom.

Wang, Y., P. Luo, and X. Li. 1996. Studies on compatibility in intergeneric crosses between Crambe abyssinica and genus Brassica. J. Sichuan Univ. Sci. (China) 33(5):590-594. 